\title{
Atemwegsprobleme durch Luftverschmutzung: Welche Kinder sind gefährdet?
}

\begin{abstract}
Ein Zusammenhang zwischen erhöhter Luftverschmutzung und allergischer Veranlagung bzw. Asthma wird immer wieder diskutiert, ein epidemiologisch einwandfreier Nachweis gelang aber noch nicht. Eine neue Studie grenzt eine Risikogruppe von Kindern ein, die bei erhöhten Schadstoffkonzentrationen Atemwegsprobleme entwickeln können.
\end{abstract}

\begin{abstract}
In Zeiten hoher Luftverschmutzung durch Auto- oder Industrieabgase steigen die Mortalitätsraten vor allem bei älteren Menschen durch kardiale oder respiratorische Komplikationen, wobei ein Zusammenhang heute als gesichert gilt. Auch für Kinder mit chronischen Atemwegserkrankungen zeigen verschiedene epidemiologische Studien einen signifikanten Zusammenhang zwischen einer sich verschlechternden Lungenfunktion und steigender Luftbelastung.
\end{abstract}

Es wird vermutet, dass speziell Kinder mit einer allergischen Veranlagung empfindlich auf erhöhte Konzentrationen z.B. von Stickstoff- und Schwefeldioxid oder Rußpartikel reagieren. Trotzdem fehlt für diese Annahme eine beweisende größere epidemiologische Studie - obwohl ein solcher $\mathrm{Zu}$ sammenhang aufgrund der Pathophysiologie des Asthma auf der Hand liegt.

\section{Verwirrende Studienlage}

Das Gegenteil ist jedoch der Fall: So ergab sich während der größeren Smogepisoden in Europa - z.B. 1952 und 1985 - kein Zusammenhang zwischen hoher Luftbelastung und gestiegener Asthmamortalität (Burney P. Lancet 1999; 353: 859-60). Untersuchungen mit Hilfe von neuen, empfindlichen Meßmethoden sowohl für die Luftbelastung wie auch für klinisch-immunologische Parameter sind daher notwendig.
Einen solchen Ansatz für den Nachweis eines möglichen Zusammenhangs zwischen Luftverschmutzung und allergischer Disposition gibt jetzt eine holländische Untersuchung, in der man die Reaktion von allergisch sensibilisierten Kindern mit der von nicht-sensibilisierten Kindern auf eine erhöhte Schadstoffbelastung der Luft verglich.

Dazu wurden eine evtl. auftretende klinische Symptomatik - z.B. Atemnot, „Wheezing"-Attacken, blockierte oder laufende Nase - sowie immunologische Daten - bronchiale Hyperreaktivität, gemessen mit dem Metacholinprovokationstest, und Bestimmung des SerumGesamt-IgEs - von insgesamt 632 Kindern über jeweils 3 Monate während dreier aufeinander folgender Winterperioden ermittelt und diese Daten in Bezug zur jeweils aktuellen Luftbelastung gesetzt.
Der Datensatz von 459 Kindern war komplett und konnte ausgewertet werden. Danach zeigten 26\% der Kinder eine bronchiale Hyperreaktivität (BHR) und ein hohes, über dem Median liegendes Gesamt-IgE, 36\% weder eine BHR noch ein erhöhtes Gesamt$\mathrm{IgE}, 15 \%$ eine $\mathrm{BHR}$ und relativ niedrige, unterhalb des Medians liegende IgEWerte und 23\% relativ hohe GesamtIgE-Konzentrationen, aber keine Anzeichen für eine BHR.

\section{Maßgeblich: der Immunstatus}

Während die Kinder der 3 letztgenannten Gruppen hinsichtlich ihrer klinischen Symptomatik von Schwankungen der Schadstoffbelastung in der Luft statistisch gesehen unbeeinflusst bleiben, ergab sich bei den Kindern mit einer erhöhten BHR und erhöhten IgE-Werten ein deutlicher Zusammenhang: Die Prävalenz von Atemwegssymptomen stieg signifikant und konzentrationsbezogen mit steigender Belastung durch Partikel, Stickstoff- oder Schwefeldioxid an. Ein Abfall der Peak-flowWerte um mehr als $10 \%$ stand in dieser Gruppe ebenfalls in einem konzentrationsabhängigen Zusammenhang mit steigender Belastung durch Teilchen mit einem Durchmesser von weniger als $10 \mu \mathrm{m}$.

\section{Risikogruppe eingegrenzt}

Über immunologische Zusammenhänge dieser Ergebnisse schweigen sich die Autoren der Studie in ihrer Diskussion aus. Sie wollen ihre Untersuchung als einen Beitrag für weitere Public-HealthStudien verstanden wissen. Ziel dabei ist, zur Einleitung gezielter Präventionsmaßnahmen die Risikogruppen einzugrenzen. Gefährdet durch „Wintersmog" sind dieser Studie zufolge Kinder mit einer erhöhten bronchialen Hyperreaktivität und erhöhtem Gesamt-IgE, die aber noch nicht zwangsläufig unter einem manifesten Asthma leiden müssen.

\section{Boezen HM, et al.}

Effects of ambient air pollution on upper and lower respiratory symptoms and peak expiratory flow in children. Lancet 1999; 353: 874-8. 